\title{
Genotyping of Foot-and-Mouth Disease Viruses Collected in Sudan Between 2009 and 2018
}

Nick Knowles ${ }^{1}$, Yazeed A. Raouf ${ }^{2}$, Jemma Wadsworth ${ }^{1}$, Abdelghani Bi-Tarif ${ }^{1}$, Ashley R. Gray $^{1}$, Mohammed Habiela ${ }^{2}$, Amira A/Almutalb ${ }^{2}$, Hanan Yousif ${ }^{2}$, Maysa Ragab², Wefag Al Fouz ${ }^{2}$, Nussiba H. Ahmed ${ }^{2}$, Inas Ibrahim ${ }^{2}$, Ahmed Almustafa ${ }^{2}$, Markos Tibbo ${ }^{3}$, Ahmad M. Almajali ${ }^{3}$, Cornelis Maanen ${ }^{4}$, Nicholas Lyons ${ }^{1}$, and Donald King ${ }^{1}$

${ }^{1}$ Pirbright Institute

${ }^{2}$ Central Veterinary Research Laboratory (CVRL) Khartoum Sudan

${ }^{3}$ Food and Agriculture Organization of the United Nations (FAO) Subregional Office for the Gulf Cooperation Council States and Yemen Abu Dhabi 62072 United Arab Emirates ${ }^{4}$ The European Commission for the Control of Foot-and-Mouth Disease (EuFMD) Food and Agriculture Organization of the United Nations (FAO) Rome Italy

August 25, 2021

\begin{abstract}
Foot-and-mouth disease (FMD) is widely distributed in Sudan where outbreaks occur on an annual basis especially during the winter months (December-February). This study aimed to increase our understanding of the epidemiological patterns of FMD in Sudan and connections to neighbouring countries by characterising the genetic sequences of FMD viruses (FMDV) collected from seven Sudanese states over a 10-year period (between 2009 and 2018). FMDV was detected in 91 of the 265 samples using an antigen-detection ELISA. Three serotypes were detected: O (46.2\%), A (34.1\%), and SAT 2 (19.8\%). Fifty-three of these samples were submitted for sequence analyses, generating sequences that were characterised as belonging to O/EA-3 $(\mathrm{n}=18)$, A/AFRICA/G-IV $(\mathrm{n}=23)$ and SAT 2/VII/Alx-12 $(\mathrm{n}=12)$ viral lineages. Phylogenetic analyses provided evidence that FMDV lineages were maintained within Sudan, and also highlighted epidemiological connections to FMD outbreaks reported in neighbouring countries in East and North Africa (such as Ethiopia and Egypt). This study motivates continued FMD surveillance in Sudan to monitor the circulating viral lineages and broader initiatives to improve our understanding of the epidemiological risks in the region.
\end{abstract}

\section{Genotyping of Foot-and-Mouth Disease Viruses Collected in Sudan Between 2009 and 2018}

Yazeed A. Raouf ${ }^{1}$, Jemma Wadsworth ${ }^{2}$, Abdelghani Bin-Tarif ${ }^{2}$, Ashley R. Gray ${ }^{2}$, Mohammed Habiela ${ }^{1}$, Ameera A. Almutalb ${ }^{1}$, Hanan Yousif ${ }^{1}$, Maysa Ragab ${ }^{1}$, Wefag Al Fouz ${ }^{1}$, Nussiba H. Ahmed ${ }^{1}$, Inas Ibrahim ${ }^{1}$, Ahmed M. Hassan ${ }^{1}$, Markos Tibbo ${ }^{3}$, Ahmad M. Almajali ${ }^{3,4}$, Cornelis van Maanen ${ }^{5}$, Nicholas A. Lyons ${ }^{2,5}$, Donald P. King ${ }^{2}$ and Nick J. Knowles ${ }^{2}$

${ }^{1}$ Central Veterinary Research Laboratory (CVRL), Khartoum, Sudan.

2 The Pirbright Institute, Ash Road, Pirbright, Woking, Surrey, GU24 0NF, United Kingdom.

${ }^{3}$ Food and Agriculture Organization of the United Nations (FAO), Subregional Office for the Gulf Cooperation Council States and Yemen, Abu Dhabi 62072, United Arab Emirates.

${ }^{4}$ Department of Veterinary Clinical Sciences, Faculty of Veterinary Medicine, Jordan University of Science and Technology, Irbid 22110, Jordan. 
${ }^{5}$ The European Commission for the Control of Foot-and-Mouth Disease (EuFMD), Food and Agriculture Organization of the United Nations (FAO), Rome, Italy.

Corresponding author: N.J. Knowles, The Pirbright Institute, Ash Road, Pirbright, Woking, Surrey, GU24 0NF, United Kingdom. Tel: + 44 (0)1483 232441; fax: + 44 (0)1483 232446; e-mail: nick.knowles@pirbright.ac.uk; OCHID iD: https://orcid.org/0000-0002-6696-1344.

\section{Keywords:}

Aphthovirus; epidemiology; foot-and-mouth disease; nucleotide sequence; Sudan

\section{Abstract}

Foot-and-mouth disease (FMD) is widely distributed in Sudan where outbreaks occur on an annual basis especially during the winter months (December-February). This study aimed to increase our understanding of the epidemiological patterns of FMD in Sudan and connections to neighbouring countries by characterising the genetic sequences of FMD viruses (FMDV) collected from seven Sudanese states over a 10-year period (between 2009 and 2018). FMDV was detected in 91 of the 265 samples using an antigen-detection ELISA. Three serotypes were detected: O (46.2\%), A (34.1\%), and SAT 2 (19.8\%). Fifty-three of these samples were submitted for sequence analyses, generating sequences that were characterised as belonging to O/EA$3(\mathrm{n}=18)$, A/AFRICA/G-IV $(\mathrm{n}=23)$ and SAT 2/VII/Alx-12 $(\mathrm{n}=12)$ viral lineages. Phylogenetic analyses provided evidence that FMDV lineages were maintained within Sudan, and also highlighted epidemiological connections to FMD outbreaks reported in neighbouring countries in East and North Africa (such as Ethiopia and Egypt). This study motivates continued FMD surveillance in Sudan to monitor the circulating viral lineages and broader initiatives to improve our understanding of the epidemiological risks in the region.

\section{1 | INTRODUCTION}

Foot-and-mouth disease (FMD) remains a worldwide major constraint to animal production and international trade. The causative agent, FMD virus (FMDV; genus Aphthovirus, family Picornaviridae), exists as seven immunologically distinct serotypes (O, A, C, Asia 1, SAT 1, SAT 2 and SAT 3) and the virus is known to infect up to 70 cloven-hoofed animal species (Grubman and Baxt, 2004). The epidemiology of FMD in Africa is complex. This is partly because the continent is resident to three out of the seven endemic FMD virus pools (Pools 4, 5 and 6; Paton et al., 2009), and six out of the seven FMD virus serotypes have been recorded (Rweyemamu et al., 2008), although serotype C has not been detected since 2004 (Paton et al., 2021). Sudan is one of the largest African countries (nearly 1.9 million $\mathrm{km}^{2}$ ) which has a resident population of more than 100 million FMD-susceptible animals. It has 30-40 million cattle, 70 million small ruminants as well as a diverse range of FMD susceptible wildlife species that are located mostly in the Southern areas or in the Dinder National Park in Eastern Sudan (Anon, 2009). Sudan is recognised as an important crossroads between sub-Saharan and Northern Africa, and between East and West Africa.

FMD has been known to be present in Sudan since 1903 (Abu Elzein, 1983) and previous studies have highlighted the contribution of FMD circulation in the country to the wider epidemiology of the disease in Africa (Bronsvoort et al., 2004; Rweyemamu et al., 2008; Ularamu et al., 2017). In sub-Saharan African countries, FMD is endemic but clinical signs are often mild or inapparent particularly in sheep and goats and outbreaks are inconsistently reported to veterinary services. Together with the costs and logistics associated with the shipment of samples, these issues result in infrequent submissions to international reference centres [such as the World Reference Laboratory for FMD (WRLFMD), Pirbright, United Kingdom] and consequent potential for biases in epidemiological inferences (Bronsvoort et al., 2004). To address these sampling and reporting issues, the Government of Sudan together with the Food and Agriculture Organization of the United Nations (FAO) and the European Commission for the Control of FMD (EuFMD) joined efforts in a Technical Corporation Programme (TCP/SUD/3401) to promote active surveillance of FMD in Sudan which was undertaken between 2012 and 2014. This project analysed more than 1500 bovine sera and more than 1000 sera from small ruminants for antibodies against FMD virus (data reported elsewhere, Raouf et al., 2016; 2017). Furthermore, epithelium samples were collected during 2013-2018 from suspected disease 
events in six Sudanese states for virus detection, serotyping and molecular characterization. Four different FMDV serotypes have been reported to circulate in Sudan (O, A, SAT 1 and SAT 2) with SAT 1 last detected in 1976 (Habiela et al., 2010a). In previous reports, type O has been detected more frequently while serotypes SAT 2 and A are found more sporadically. The aim of this current study is to improve our understanding of the epidemiological patterns of FMD in Sudan and connections to neighbouring countries through characterising FMDV in clinical samples.

\section{2 | MATERIALS AND METHODS}

\section{1 | Clinical samples}

During passive surveillance undertaken in the period 2009-2018, epithelium tissues $(n=265)$ were collected from suspected cases of FMD in 10 Sudanese states: Khartoum, River Nile, Gezira, Al Qadarif, White Nile, Blue Nile, North Kordofan, Kassala, North and South Darfur. Each year between 15 and 30 samples were collected except for 2013 and 2014 when more than 100 samples were collected.

\section{2 | FMDV detection and serotyping}

All samples were screened for the presence of FMDV antigen either using the indirect sandwich ELISA kit (WRLFMD; Roeder and Le Blanc Smith, 1987) or using the monoclonal antibody based antigen detection and serotyping ELISA kit (IZSLER Biotech, Brescia, Italy; Grazioli et al., 2012). A subset of these FMDV positive samples $(\mathrm{n}=53)$ were submitted to the WRLFMD (at the Pirbright Institute, Surrey, UK) where they were also tested by real time RT-PCR (Callahan et al., 2002; Shaw et al., 2007) and virus isolation using primary bovine thyroid and IB-RS-2 cells as previously described (Snowdon, 1966; de Castro, 1964). Samples in which FMDV was identified $(\mathrm{n}=91)$ are listed in Table 1 together with associated metadata.

\section{3 | Sequencing and phylogenetic analysis}

VP1 sequences were generated using previously described methods (Knowles et al., 2016). Complete VP1 nucleotide sequences were aligned using BioEdit 7.0.5.3 (Hall, 1999) and Clustal W 1.83 (Thompson et al., 1994). Optimal nucleotide substitution models were computed for each serotype using MEGA 7 (Kumar et al., 2016). The maximum likelihood algorithm was used to construct phylogenetic trees employing using MEGA 7. One thousand bootstrap pseudo-replicates were used to assess branching confidence.

\section{3 | RESULTS}

\section{1 | FMD positive samples}

All 265 samples collected during this study (2009-2018) were of cattle origin. Approximately one third of these samples $(91 / 265 ; 34.3 \%)$ were successfully serotyped using antigen detection ELISA. Most of the typed samples were collected during the winter months $(62 / 91 ; 68.1 \%)$ : comprising $27 / 91(29.7 \%)$ in December, 14/91 in January (15.4\%), and 20/91 in February (22.0\%). This compares to lower numbers of FMDV positive samples collected in November $(1 / 91 ; 1.1 \%)$, March $(17 / 91 ; 18.7 \%)$ and April $(6 / 91 ; 6.6 \%)$. Only $6.6 \%$ (6/91) of the typed samples were collected between May and October.

The geographical distribution of FMDV positive samples collected in Sudan between 2009 and 2018 is described in Table 1 and Figure 1. FMD virus was detected in 91 samples which were also serotyped. Disease events comprised three serotypes; O (42/91; 46.2\%), SAT $2(18 / 91 ; 19.8 \%)$ and A $(31 / 91 ; 34.1 \%)$. FMD type $\mathrm{O}$ disease events extended over almost the entire reported period (Figure 1), apart from 2018, while serotype A was detected in 5 years $(2011,2013,2014,2015$, and 2018) and serotype SAT 2 was detected in 4 years (2010, 2013, 2014 and 2017). Serotype A was detected in the Central States of Khartoum and Gezira. In comparison, serotypes O and SAT 2 had a wider geographical distribution (Figure 1). Serotype $\mathrm{O}$ was detected along the Nile basin, from the White Nile State in the South to Khartoum and Gezira in Central Sudan and up to the Northern State in Northern Sudan. Serotype SAT 2 was detected in Central Sudan at Khartoum, Gezira and North Kordofan and in addition in one South Eastern State (Blue Nile) and one Eastern Border State (Al Qadarif). 


\section{2 | Phylogenetic analysis}

All FMDV positive samples generated a single VP1 sequence apart from SUD/7/2017 where a mixture of $\mathrm{O}$ and SAT 2 were recovered. The most appropriate nucleotide substitution models for each serotype were found to be the Tamura-Nei (TN-93) model, gamma distributed with invariant sites (G+I) (type O and type SAT 2); and the Hasegawa-Kishino-Yano (HKY) model, G+I (type A).

\section{3 | Serotype O}

All eighteen serotype $\mathrm{O}$ viruses that were genotyped during this study fell within the O/EA-3 topotype (Figure 2). These sequences represent at least four distinct clades; three of which occurred during the same period (2009-2011), while a fourth cluster contained more recent isolates from 2013, 2016 and 2017. Nucleotide identities within clusters were mostly $>95 \%$, in contrast to sequence differences between clusters of between $5 \%$ and $7 \%$. The first cluster contained isolate SUD/11/2011 which was related to other older Sudanese FMDVs collected between 2004 and 2008, with Nigerian viruses collected between 2007 and 2014 and two Cameroon viruses collected in 2010. The second cluster contained three isolates; SUD/1/2009, SUD/1/2010, and SUD/2/2010 related to FMDV sequences from Sudan in 2008, while a third cluster contained five identical sequences for FMDV isolates collected in 2012, one collected a year earlier (O/SUD/9/2011) as well as samples from Eritrea (2011) and Egypt (2012). The final clade comprised seven Sudanese FMDV isolates collected in 2016/17 and an earlier sample from 2013 (O/SUD/4/2013). This clade also contained Egyptian isolates that are part of a larger temporal phylogenetic cluster representative of O/EA-3 outbreaks reported in Egypt, Israel and Palestine.

\subsection{Serotype A}

Twenty-three serotype A viruses belonging to the A/AFRICA/G-IV lineage were genotyped (Figure 3) during this study. For Sudanese FMD viruses, collected during 2018, sequences belonged to two genetic clades (12.8-14.6\% nt difference), with older sequences represented in ancestral clusters. One of these contemporary clusters containing A/SUD/6-9/2018 also contained sequences for FMD viruses collected during 2015-17 in Egypt. At the common root of both clusters were sequences for FMDVs collected during 2006 from Sudan and Eritrea.

\section{5 | Serotype SAT 2}

Twelve serotype SAT 2 viruses within the SAT 2 topotype VII, distributed between 2010-2017 were characterised (Figure 4). All of these Sudanese sequences belonged to the Alx-12 lineage which also contains sequences from FMD viruses collected from other countries including Egypt 2014 and 2017. Older Sudanese viruses (from 1977 and 2007-08) belonged to topotype XIII and were related to viruses from Ethiopia.

\section{4 | DISCUSSION}

During the study period (2009-2018), clinical cases in cattle were observed year-round over a wide geographical distribution with relatively more cases to be reported in the winter months (December-February). So far, four FMDV serotypes have been reported in Sudan: O, A, SAT 1 and SAT 2 (Abu Elzein, 1983), although no cases due to the SAT 1 serotype were detected in this study or have been recorded in Sudan since 1976 (Abu Elzein and Crowther, 1979). The low incidence of SAT1 in Sudan is supported by serological data for SAT1-specific antibodies in comparison to serotypes O and A (Raouf et al., 2009; Habiela et al., 2010a). Serotype O was the most frequently detected; findings which are also supported by recent serological data (Raouf et al., 2016) showing higher prevalence of serotype O specific antibodies (60.2\%) compared to serotype A (30.0\%) and serotype SAT 2 (12.3\%). Nucleotide distances of more than $15 \%$ (for serotypes O and A) or 20\% (for serotypes SAT 1 and SAT 2) are used to classify isolates into different topotypes (Samuel and Knowles, 2001; Knowles and Samuel, 2003), while nucleotide differences of between $5 \%$ and 15\% indicate distinct virus lineages (Bronsvoort et al., 2004). The Sudanese sequences reported here differed from the prototype strains of the relevant topotypes (Knowles et al., 2016) by 7.7-12.1\% (O/SUD/2/86), 13.8-16\% (O/ETH/3/2004), 15.2-17.8\% (O/ETH/2/2006), 12.5-15.5\% (O/ETH/1/2007), 13.4-16\% (A/SUD/3/77), 
7.4-9.9\% (SAT2/SAU/6/2000) and 11.9-14.4\% (SAT2/CAR/8/2005). These differences indicated the continued divergence of virus lineages within each topotype.

Serological findings from 2013 (Raouf et al., 2016) demonstrate that serotype O is present along the Nile basin up to Khartoum and in Western, Eastern and Northern Sudan. A previous study (Habiela et al., 2010b) highlighted that within-country circulation is an important mechanism by which serotype $\mathrm{O}$ virus is maintained in Sudan. New FMDV sequence data generated here continues to demonstrate that serotype O is maintained in the country over successive years without any requirement for the introduction of viruses via transboundary pathways. This point is supported by the presence of at least two viral lineages within the phylogenetic tree that only contain sequences from Sudan (2004-2011 and 2008-2010). The three lineages containing Sudanese and Egyptian sequences are indicative of the northerly spread of FMDV O/EA-3 from Sudan, which based on the dates occurred as separate independent events during 2011-2012, 2013-2016 and 2016-2017. One of these clades also contains a sequence from Eritrea, highlighting other potential transboundary transmission pathways to other countries in the neighbourhood.

Data for serotype A sequence also support the concept that FMD virus lineages are maintained solely within Sudan. The phylogenetic tree contains two lineages that comprise sequences only from Sudan collected from different years (2013-2014 and 2018). In common with the serotype O data, there was also evidence for the northerly spread of A/AFRICA/G-IV into Egypt (during 2011-2012). Epidemiological links to FMD cases in Ethiopia are highlighted in a second clade that contained Egyptian sequences (from 2015-2017) and more recent sequences collected from Sudan (from 2018), where the Ethiopian sequence (A/ETH/19/2015) pre-dated those for the Sudanese samples. More broadly, the serotype A (and serotype O) phylogenetic trees highlight the relationship between recent sequences for FMD viruses collected in Ethiopia (Gizaw et al., 2020) and contemporary data from Sudan. For both serotypes, there are examples where Sudanese sequences have a more basal ancestral location in the tree; for example, the relationships between O/Sudan/2012 and O/Ethiopia/2018-19 and A/Sudan/2013-14 and A/Ethiopia/2018-19, respectively. These results indicate that these FMD viruses have spread from Sudan into Ethiopia; however, this interpretation of the data should be treated cautiously in view of the likely high number of unsampled FMD cases in both countries where the sampling density is very low and susceptible livestock populations are high.

Serotype SAT 2 was the last FMDV serotype to be detected in Sudan in 1976 (Abu Elzein and Crowther, 1979) and 18 Sudanese isolates of serotype SAT 2 were detected during four of the years during this study. All of these sequences from 2010-2017 were characterised as belonging to the SAT 2/VII/Alx-12 lineage that has spread widely in the region to cause FMD outbreaks in countries such as Egypt, Palestine and Israel. In common with serotypes $\mathrm{O}$ and $\mathrm{A}$, sequences for viruses from this lineage collected from Sudan are earlier compared to those for viruses sampled in Ethiopia. The SAT 2/VII/Alx-12 lineage is distinct to earlier viral clades detected in Sudan such as those that have spread previously from East Africa into West Africa (Habiela et al., 2010b; Ularamu et al., 2017: highlighted by presence of SAT2/SUD/1/2007 in the phylogenetic tree).

No previous reports have described clinical FMD in Sudan in species other than cattle (Abu Elzein, 1983; Raouf et al., 2010; Habiela et al., 2010a). Recent serological studies suggested a relatively low seroprevalence of non-structural protein antibodies among small ruminants at around $14 \%$, except in Khartoum (Habiela et al., 2009) and Blue Nile State (Raouf, 2015; Raouf et al., 2017). This may indicate a varied role of sheep in the epidemiology of FMD. FMD infection in wild ruminants in Sudan has not been investigated.

In summary, FMD infection in Sudan remains regionally significant and this study highlights the epidemiological connections between FMDV sequences collected in Sudan and neighbouring countries such as Ethiopia and Egypt. Due to incomplete and convenience-biased sampling, it should be recognised that there are limitations in our understanding of the transboundary connectivity in the region and the sequences reported here provide only a crude snapshot survey of the underlying FMD transmission events. Recognition of border areas as particularly risky hotspots for the introduction of FMD viruses is of high importance in developing a risk-based control strategy especially when resources are limited. In this context, continued studies are warranted to improve our understanding of FMD epidemiology in Sudan and risk-pathways in the region. 


\section{ACKNOWLEDGEMENTS}

The work in Sudan was supported by the FAO Technical Corporation Programme, TCP/SUD/3401. The sequencing work at the WRLFMD was supported by the Department for Environment, Food and Rural Affairs (Defra), UK under research grant no. SE2944. The work of the WRLFMD is supported with funding provided from the European Union (via a contract from EuFMD, Rome). The views expressed herein can in no way be taken to reflect the official opinion of the European Union. The Pirbright Institute receives grant-aided support from the Biotechnology and Biological Sciences Research Council of the United Kingdom (projects BBS/E/I/00007035 and BBS/E/I/00007036). We would like to thank Dr. Antonello Di Nardo for help in constructing the map in Figure 1.

\section{CONFLICT OF INTEREST}

The authors declare no commercial or financial conflict of interest.

\section{ETHICAL STATEMENT}

The authors confirm that the ethical policies of the journal, as noted on the journal's author guidelines page, have been adhered to. No ethical approval was required as this this study did not involve any experimental animal protocols.

\section{DATA AVAILABILITY STATEMENT}

All nucleotide sequences generated were submitted to GenBank and accession numbers can be found in Table 1.

\section{REFERENCES}

Abu Elzein, E. M. E. (1983). Foot and mouth disease in the Sudan.Revue scientifique et technique de l'Office International des Epizooties , 2, 177-188. https://doi.org/10.20506/rst.2.1.106

Abu Elzein E. M. E., \& Crowther J. R. (1979). Serological comparison of a type SAT2 foot-and-mouth disease virus isolate from Sudan with other type SAT2 strains. Bulletin of Animal Health and Production in Africa , 27, 245-248.

Anonymous (2009). Sudan's Fourth National Report to the Convention on Biological Diversity. The Higher Council for Environment and Natural Resources (HCENR), Ministry of Environment and Physical Development, Government of the Republic of Sudan, 77 pp. https://www.cbd.int/doc/world/sd/sd-nr-04-en.pdf

Bronsvoort, B. M. de C., Radford, A. D., Tanya, I. V. N., Nfon, C., Kitching, R. P., \& Morgan K. L. (2004). Molecular epidemiology of foot-and-mouth disease viruses in the Adamawa Province of Cameroon.Journal of Clinical Microbiology , 42, 2186-2196. https://doi.org/10.1128/JCM.42.5.2186-2196.2004

Callahan, J. D., Brown, F., Osorio, F. A., Sur, J. H., Kramer, E., Long, G. W., Lubroth, J., Ellis, S. J., Shoulars, K. S., Gaffney, K. L., Rock, D. L., \& Nelson, W. M. (2002). Use of a portable real-time reverse transcriptase-polymerase chain reaction assay for rapid detection of footand-mouth disease virus. Journal of the American Veterinary Medical Association , 220, 1636-1642. https://doi.org/10.2460/javma.2002.220.1636.

de Castro, M. P. (1964). Behavior of foot-and-mouth disease virus in cell culture: susceptibility of the IB-RS-2 swine cell line.Arquivos do Instituto Biologico Sao Paulo , 31, 63-78.

Gizaw, D., Tesfaye, Y., Wood, B. A., Di Nardo, A., Shegu, D., Muluneh, A., Bilata, T., Belayneh, R., Fentie, A., Asgdome, H., Sombo, M., Rufael, T., Tadesse Woldemariyam, F., Khan, F., Yami, M., Gelaye, E., Wadsworth, J., Knowles, N. J., \& King, D. P. (2020). Molecular characterization of foot-and-mouth disease viruses circulating in Ethiopia between 2008 and 2019. Transboundary and Emerging Diseases , 67, 2983-2992. https://doi.org/10.1111/tbed.13675. Erratum in: Transboundary and Emerging Diseases , 68, 2636. https://doi.org/10.1111/tbed.14167 
Grazioli, S., Ferris, N., Dho, G., Spagnoli, E., \& Brocchi, E. (2012). Ready-to-use ELISA kit for FMDV diagnosis and serotyping tailored for Africa. Session of the Research Group of the Standing Technical Committee of EuFMD, Jerez, Spain, 29 Oct-31 Oct 2012.

Grubman, M. J., \& Baxt, B. (2004). Foot-and-mouth disease.Clinical Microbiology Reviews , 17, 465-493. https://doi.org/10.1128/CMR.17.2.465-493.2004

Habiela, M., Raouf, Y. A., \& Nur Eldin, H., (2009). Sero-survey of anti-foot and mouth disease virus antibodies in sheep and goats in Khartoum state, Sudan. The Sudan Journal of Veterinary Research, 24, 61-64.

Habiela, M., Alamin, M. A. G., Raouf, Y. A., \& Ali, Y. A. (2010a). Epizootiological study of foot and mouth disease in the Sudan: the situation after two decades. Veterinarski Arhiv , 80, 11-26.

Habiela, M., N. P. Ferris, G. H. Hutchings, J. Wadsworth, S. M. Reid, M. Madi, K. Ebert, K. J. Sumption, N. J. Knowles, D. P. King, \& D. J. Paton, (2010b). Molecular characterization of foot-and-mouth disease viruses collected from Sudan. Transboundary and Emerging Diseases , 57, 305-314. https://doi.org/10.1111/j.18651682.2010.01151.x

Hall, T. A. (1999). BioEdit: a user-friendly biological sequence alignment editor and analysis program for Windows 95/98/NT.Nucleic Acids Symposium Series , 41, 95-98.

Hall, M. D., Knowles, N. J., Wadsworth, J., Rambaut, A., \& Woolhouse, M. E. (2013). Reconstructing geographical movements and host species transitions of foot-and-mouth disease virus serotype SAT 2. mBio , 4, e00591-13. https://doi.org/10.1128/mBio.00591-13

Knowles, N. J., \& Samuel A. R. (2003). Molecular epidemiology of foot-and-mouth disease virus. Virus Research , 91, 65-80. https://doi.org/10.1016/s0168-1702(02)00260-5

Knowles N.J., Wadsworth J., Bachanek-Bankowska K., \& King D.P. (2016). VP1 sequencing protocol for foot and mouth disease virus molecular epidemiology. Revue scientifique et technique de l'Office International des Epizooties , 35, 741-755. https://doi.org/10.20506/rst.35.3.2565

Kumar, S., Stecher, G., \& Tamura, K. (2016). MEGA7: Molecular Evolutionary Genetics Analysis Version 7.0 for Bigger Datasets.Molecular Biology and Evolution , 33, 1870-1874. https://doi.org/10.1093/molbev/msw054

Paton, D.J., Sumption, K.J., \& Charleston, B. (2009). Options for control of foot-and-mouth disease: knowledge, capability and policy.Philosophical Transactions of the Royal Society B: Biological Sciences , 364, 2657-2667. https://doi.org/10.1098/rstb.2009.0100

Paton, D. J., Di Nardo, A., Knowles, N. J., Wadsworth, J., Pituco, E. M., Cosivi, O., Rivera, A. M., Bakkali Kassimi, L., Brocchi, E., de Clercq, K., Carrillo, C., Maree, F. F., Singh, R. K., Vosloo, W., Park, M.-K., Sumption, K. J., Ludi, A. B., \& King, D. P. (2021). The history of foot-and-mouth disease virus serotype C: the first known extinct serotype? Virus Evolution 7, 2057-1577. https://doi.org/10.1093/ve/veab009

Raouf, Y. A. (2015). Some observations on the comparative performance of two 3-ABC ELISAs in an area of endemicity. Bulletin of Animal Health and Production in Africa, 63, 129-138.

Raouf Y. A., Ali, B. H., Khair, S. M., \& Amin, A. M. (2009). The Prevalence of antibodies against SAT1 foot-and-mouth disease virus in cattle in Khartoum State: epidemiological significance. Bulletin of Animal Health and Production in Africa , 57, 339-347. https://doi.org/10.4314/bahpa.v57i4.51680

Raouf, Y. A., Ali, B., Amin, M. A. I., \& Al Shallali, A. M. (2010). Laboratory investigation of three outbreaks of foot-and-mouth disease at central Sudan and the disease type situation. Bulletin of Animal Health and Production in Africa, 58, 308-314.

Raouf, Y. A., Tamador, M. A. A., Nahid, A. I., \& Shaza, M. (2012). A survey for antibodies against current infection of foot-and-mouth disease virus in Sudanese cattle, sheep and goats using neutralization 
test. Bulletin of Animal Health and Production in Africa, 60, 351-358.

Raouf, Y. A., Yousif, H., Almutlab, A. A., Hassan, A. A., Ibra, A., Tibbo, M., \& Al-Majali, A. (2016). Sero-epidemiology of foot-and-mouth disease in Sudan. Bulletin of Animal Health and Production in Africa , 64, 443-451.

Raouf, Y. A., Yousif, H., Almutlab, A. A., Hassan, A. A., Al-Majali, A. \& Tibbo, M. (2017). Role of small ruminants in the epidemiology of foot-and-mouth disease in Sudan. Bulletin of Animal Health and Production in Africa, 65, 145-156.

Roeder, P. L., \& Le Blanc Smith, P. M. (1987). Detection and typing of foot-and-mouth disease virus by enzyme-linked immunosorbent assay: a sensitive, rapid and reliable technique for primary diagnosis. Research in Veterinary Science , 43, 225-232. https://doi.org/10.1016/S0034-5288(18)30778-1

Rweyemamu, M., Roeder, P., Mackay, D., Sumption, K., Brownlie, J., Leforban, Y., Valarcher, J. F., Knowles, N. J., \& Saraiva, V. (2008). Epidemiological patterns of foot-and-mouth disease worldwide.Transboundary and Emerging Diseases , 55, 57-72. https://doi.org/10.1111/j.1865-1682.2007.01013.x

Samuel, A. R., \& Knowles, N. J. (2001). Foot-and-mouth disease type O viruses exhibit genetically and geographically distinct evolutionary lineages (topotypes). Journal of General Virology, 82, 609-621. https://doi.org/10.1099/0022-1317-82-3-609

Shaw, A. E., Reid, S. M., Ebert, K., Hutchings, G. H., Ferris, N. P., \& King, D. P. (2007). Implementation of a one-step real-time RT-PCR protocol for diagnosis of foot-and-mouth disease. Journal of Virological Methods , 143, 81-85. https://doi.org/10.1016/j.jviromet.2007.02.009.

Snowdon WA. (1966). Growth of foot-and mouth disease virus in monolayer cultures of calf thyroid cells. Nature , 210(5040), 1079-1080. https://doi.org/10.1038/2101079a0.

Thompson, J. D., Higgins, D. G., \& Gibson, T. J. (1994). CLUSTAL W: improving the sensitivity of progressive multiple sequence alignment through sequence weighting, position-specific gap penalties and weight matrix choice. Nucleic Acids Research , 22, 4673-4680. https://doi.org/10.1093/nar/22.22.4673

Ularamu, H. G., Ibu, J. O., Wood, B. A., Abenga, J. N., Lazarus, D. D., Wungak, Y. S., Knowles, N. J., Wadsworth, J., Mioulet, V., King, D. P., Shamaki, D., \& Adah, M. I. (2017). Characterization of foot-andmouth disease viruses collected in Nigeria between 2007 and 2014: evidence for epidemiological links between West and East Africa. Transboundary and Emerging Diseases , 63, 1-10. https://doi.org/10.1111/tbed.12584

\section{FIGURE LEGENDS}

FIGURE 1. Geographical distribution of FMDV outbreaks in Sudan 2009-2018. Map represents Sudanese provinces where the intensity of the colour reflects on cattle density (FAO). Circles define numbers of samples that were serotyped using antigen detection ELISA (Red: serotype O; blue: serotype A; purple: serotype SAT 2).

FIGURE 2. Midpoint-rooted maximum likelihood tree showing the relationships between the VP1 sequences of the 2009-2017 serotype O viruses from Sudan (indicated with red diamonds) and other contemporary and reference viruses. Bootstrap values of $70 \%$ and above are shown. ${ }^{*}$, Reference number not assigned by the WRLFMD.

FIGURE 3. Midpoint-rooted maximum likelihood tree showing the relationships between the VP1 sequences of the 2011-2018 serotype A viruses from Sudan (indicated with blue diamonds) and other contemporary and reference viruses. Bootstrap values of $70 \%$ and above are shown. ${ }^{*}$, Reference number not assigned by the WRLFMD.

FIGURE 4. Midpoint-rooted maximum likelihood tree showing the relationships between the VP1 sequences of the 2010-2017 serotype SAT 2 viruses from Sudan (indicated with purple diamonds) and other contempo- 
rary and reference viruses. Bootstrap values of $70 \%$ and above are shown. ${ }^{*}$, Reference number not assigned by the WRLFMD.

\section{Hosted file}

Fig 1.pptx available at https://authorea.com/users/431680/articles/535185-genotyping-offoot-and-mouth-disease-viruses-collected-in-sudan-between-2009-and-2018

\section{Hosted file}

Fig 2.pptx available at https://authorea.com/users/431680/articles/535185-genotyping-offoot-and-mouth-disease-viruses-collected-in-sudan-between-2009-and-2018

\section{Hosted file}

Fig 3.pptx available at https://authorea.com/users/431680/articles/535185-genotyping-offoot-and-mouth-disease-viruses-collected-in-sudan-between-2009-and-2018

\section{Hosted file}

Fig 4.pptx available at https://authorea.com/users/431680/articles/535185-genotyping-offoot-and-mouth-disease-viruses-collected-in-sudan-between-2009-and-2018

\section{Hosted file}

Table 1.docx available at https://authorea.com/users/431680/articles/535185-genotyping-offoot-and-mouth-disease-viruses-collected-in-sudan-between-2009-and-2018 